\title{
Rationale, design and methods of VA-BRAVE: a randomized comparative effectiveness trial of two formulations of buprenorphine for treatment of opioid use disorder in veterans
}

Ismene Petrakis ${ }^{1,2^{*}} \mathbb{0}$, Sandra A. Springer ${ }^{3,4+}$, Cynthia Davis ${ }^{5,6}$, Elizabeth Ralevski ${ }^{2,4}$, Lucy Gu ${ }^{1,2}$, Robert Lew ${ }^{7,8}$, John Hermos ${ }^{9,10}$, Melynn Nuite, Adam J. Gordon ${ }^{11,12}$, Thomas R. Kosten ${ }^{13,14}$, Edward V. Nunes ${ }^{15}$, Robert Rosenheck ${ }^{1,2}$, Andrew J. Saxon ${ }^{16,17}$, Robert Swift ${ }^{18,19}$, Alexa Goldberg ${ }^{20}$, Robert Ringer ${ }^{20}$ and Ryan Ferguson ${ }^{5,8}$

\begin{abstract}
Background: To address the US opioid epidemic, there is an urgent clinical need to provide persons with opioid use disorder (OUD) with effective medication treatments for OUD (MOUD). Formulations of sublingual buprenorphine/ naloxone (SL-BUP/NLX) are considered the standard of care for OUD including within the Veterans Healthcare Administration (VHA). However, poor retention on MOUD undermines its effectiveness. Long-acting injectable monthly buprenorphine (INJ-BUP) (e.g., Sublocade ${ }^{\circledR}$ ) has the potential to improve retention and therefore reduce opioid use and overdose. Designing and conducting studies for OUD pose unique challenges. The strategies and solutions to some of these considerations in designing Cooperative Studies Program (CSP) 2014, Buprenorphine for Treating Opioid Use Disorder in Veterans (VA-BRAVE), a randomized, 20-site, clinical effectiveness trial comparing INJ-BUP to SLBUP/NLX conducted within the VHA may provide valuable guidance for others confronted with similar investigation challenges.
\end{abstract}

Methods: This 52-week, parallel group, open-label, randomized controlled trial (RCT) evaluates the comparative effectiveness of two current FDA-approved formulations of buprenorphine: (1) daily SL-BUP/NLX vs. (2) monthly (28day) INJ-BUP for Veterans with moderate to severe OUD $(n=952)$. The primary outcomes are (1) retention in MOUD and (2) opioid abstinence. Secondary outcomes include measures of other drug use, psychiatric symptoms, medical outcomes including prevalence rates of HIV, hepatitis B and C as well as social outcomes (housing instability, criminal justice involvement), service utilization and cost-effectiveness. Special considerations in conducting a comparative effectiveness trial with this population and during COVID-19 pandemic were also included.

Discussion: The evaluation of the extended-release formulation of buprenorphine compared to the standard sublingual formulation in real-world VHA settings is of paramount importance in addressing the opioid epidemic. The extent to which this new treatment facilitates retention, decreases opioid use, and prevents severe sequelae of OUD

\footnotetext{
*Correspondence: Ismene.Petrakis@yale.edu

${ }^{\dagger}$ Ismene Petrakis and Sandra A. Springer are co-first authors

${ }^{1}$ Department of Psychiatry, Veterans Affairs Connecticut Healthcare

System, West Haven, CT, USA

Full list of author information is available at the end of the article
} permits use, sharing, adaptation, distribution and reproduction in any medium or format, as long as you give appropriate credit to the original author(s) and the source, provide a link to the Creative Commons licence, and indicate if changes were made. The images or other third party material in this article are included in the article's Creative Commons licence, unless indicated otherwise in a credit line to the material. If material is not included in the article's Creative Commons licence and your intended use is not permitted by statutory regulation or exceeds the permitted use, you will need to obtain permission directly from the copyright holder. To view a copy of this licence, visit http://creativecommons.org/licenses/by/4.0/. The Creative Commons Public Domain Dedication waiver (http://creativeco mmons.org/publicdomain/zero/1.0/) applies to the data made available in this article, unless otherwise stated in a credit line to the data. 
has not been studied in any long-term trial to date. Positive findings in this trial could lead to widespread adoption of MOUD, and, if proven superior INJ-BUP, by clinicians throughout the VHA and beyond. This treatment has the potential to reduce opioid use among Veterans, improve medical, psychological, and social outcomes, and save lives at justifiable cost.

Trial registration Registered at Clinicaltrials.gov NCT04375033

Keywords: Opioid use disorder, Buprenorphine, Comparative effectiveness trial, Veterans, Injectable

\section{Background}

There are many challenges and considerations to designing and conducting comparative effectiveness studies for opioid use disorder (OUD). The strategies and solutions to some of these considerations in the design of a multi-site, national comparative effectiveness trial may provide valuable guidance for others confronted with similar challenges. VA Cooperative Studies Program \#2014 (CSP \#2014) is the first long-term, direct effectiveness comparison trial of monthly injectable formulation of buprenorphine (INJ-BUP) to daily formulation of buprenorphine + naloxone (SL-BUP/NLX) and the first to be conducted in the Veterans Healthcare Administration (VHA) system among Veterans with OUD.

In October 2017, the US declared that the rise in heroin and fentanyl use [1,2], and dramatic increase of opioid overdose deaths [3], including among Veterans [4], was a national epidemic and public health emergency $[5,6]$. From October 2018-2019, over 47,000 individuals died of opioid overdoses in the United States [7, 8]. Early data emerging in 2020 suggest that overdose deaths continue to climb during the COVID-19 pandemic [9].

The number of individuals diagnosed with OUD also has increased with the rise in opioid misuse. It is estimated there were 2.1 million Americans diagnosed with OUD in 2018 [10]. The number of Veterans diagnosed with OUD who are seeking treatment from the VHA has also dramatically increased in the past few years, similar to that seen in the general community. There was a $131 \%$ increase in OUD diagnoses from 2001 (27,840 cases of OUD) to 2015 (64,373 cases), with 69,142 Veterans diagnosed with OUD in fiscal year 2017 and now over 80,000 diagnosed with OUD $[5,11]$. As in the general population, OUD among Veterans is associated with housing instability, mental health diagnoses, suicide, and criminal justice involvement [12].

The most effective treatment for OUD are medications of three types: the full opioid agonist methadone, the partial agonist buprenorphine, and the antagonist naltrexone in extended-release injectable formulation (XR-NTX) [13-16]. These medications have been shown to reduce relapse to opioid use, overdose, HIV and HCV transmission, and improve other health outcomes [17, 18]. Overall, clinic-based daily SL-BUP/NLX treatment has become the treatment of choice for OUD because it has a favorable safety profile, can be administered in community settings that include primary care provider offices and does not require detoxification prior to initiation [14]. Clinic-based treatment with SL-BUP/NLX is the standard of care for OUD within the VHA [11].

Despite evidence that daily SL-BUP/NLX is effective, it is discontinued at high rates, undermining its effectiveness. Several reviews show that buprenorphine retention at 6 months is estimated at less than $40 \%[19,20]$. A 2020 systematic review reported retention rates falling from $58 \%$ at 6 months to $38.4 \%$ at 3 years [21, 22] .In an earlier study with a Veteran population, retention rates fell from $61.6 \%$ at 1 year to $31.83 \%$ at 3 years [22]. More recently, data from the VA Center of Excellence in Substance Use Disorder Treatment and Education (unpublished, 2017) shows only $35 \%$ of Veterans were retained in SL-BUP/NLX treatment at 12 months after initiation; with the highest dropout rate (almost 25\%) within the first 30 days.

Long-term opioid abstinence is generally achieved through long term treatment with medications for opioid use disorder (MOUD), including SL-BUP/NLX [23, 24], and there is high risk for relapse and overdose particularly during the first 30 days after discontinuation [25]. Maximizing retention is thus of great clinical importance, as good retention is associated with lower mortality rates, lower emergency room utilization [26], and improvement in domains including risk for and treatment of HIV [27, 28].

Injectable buprenorphine (e.g., Sublocade ${ }^{\circledR}$ ) is a monthly alternative to the daily form of SL-BUP/NLX for treatment of persons with moderate to severe OUD. The monthly formulation provides a steady plasma concentration and thus may be more favorable for persons who have difficulty adhering to daily SL-BUP/NLX. Sublocade ${ }^{\circledR}$ has already been shown to be well tolerated and both available doses are associated with a higher percentage of abstinence $(41.3 \% ; 42.7 \%)$ compared to placebo (5.0\%) [29]. Data presented by Indivior pharmaceutical company, the manufacturer of Sublocade ${ }^{\circledR}$, show it was associated with retention in treatment at $60 \%$ at 6 months and 12 months [29] and in their final published trial data in Lancet 2019 [29] they report an overall retention rate 
as $60 \%$ in the 24 week trial. While it has not yet been directly compared to SL-BUP/NLX, a retrospective chart review comparing retention between the 2 formulations showed a significantly higher number of visits for those on Sublocade ${ }^{\circledR}$ vs. sublingual buprenorphine, but no significant difference in retention in 6 months [30].

Therefore, we designed CSP \#2014 "Buprenorphine for Treating Opioid Use Disorder in Veterans (VA-BRAVE)" (Fig. 1), a 52-week randomized controlled study to compare the effectiveness of the long acting monthly injectable formulation of buprenorphine versus the standard of care sublingual formulation, administered as part of routine outpatient care to 952 Veterans across 20 sites in the VHA. There are 2 co-primary outcomes and the study was designed to determine if long-acting injectable buprenorphine is superior to the sublingual form in retention on medication and abstinence from opioids. In addition to the study design and methods proposed to conduct this clinical trial, this manuscript describes the practical, safety and ethical considerations of working with participants who have OUD, selection of the outcomes, and duration of the trial among other issues. We discuss considerations for special sub-populations and events within the study population including pregnancy, incarceration, new onset infectious disease and overdose. Lastly, before the trial was initiated, a worldwide pandemic with COVID-19 effectively interrupted clinical care nationally; considerations on how to proceed while protecting participant and staff safety were developed and are reviewed.

\section{Methods}

\section{Study population}

The eligibility criteria to participate in this trial (Table 1) are deliberately broad to maximize the inclusion of Veterans in order to assess the co-primary outcomes (retention in MOUD and opioid abstinence) and to mirror 'real world' patients with OUD who present for MOUD

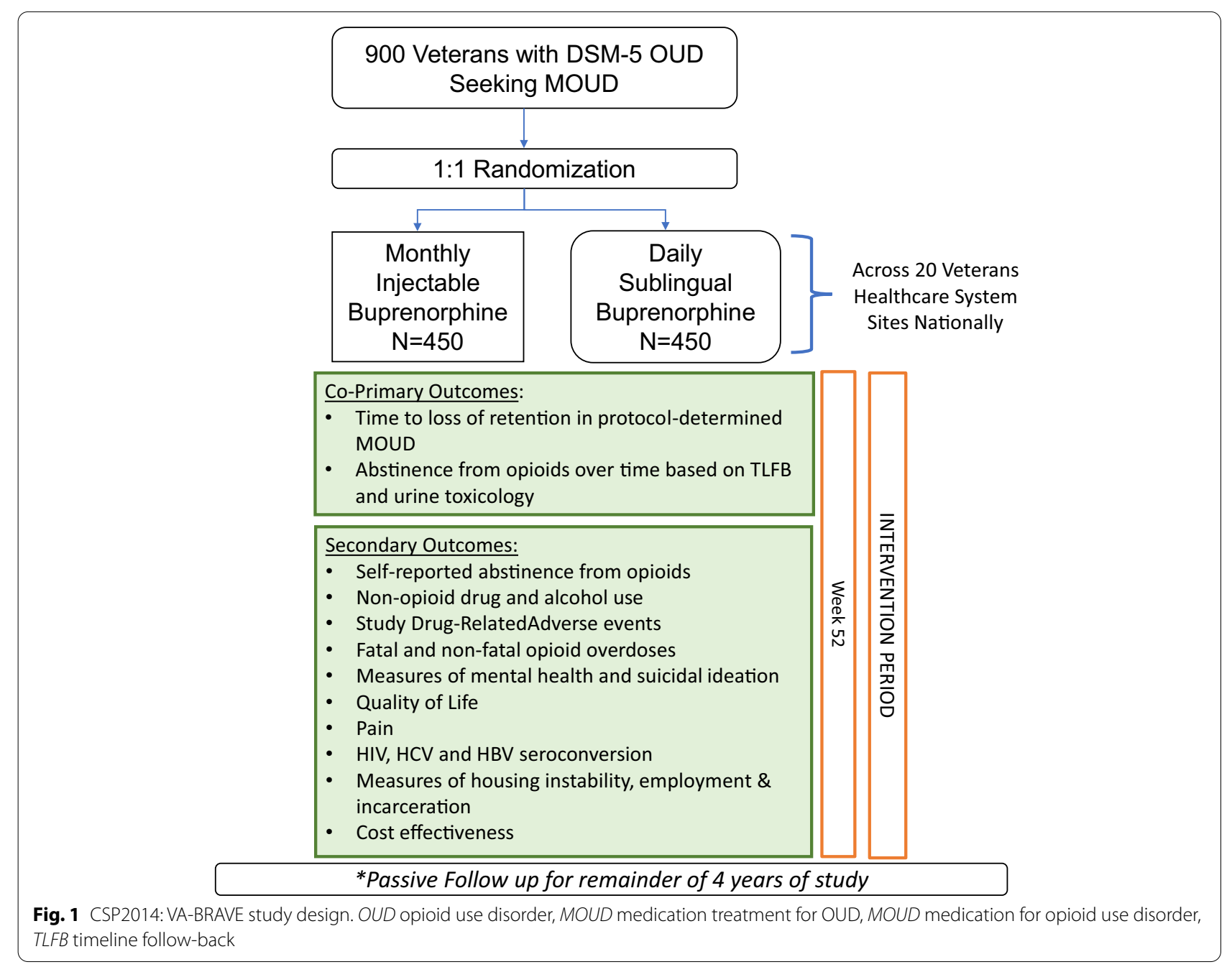


Table 1 CSP\#2014:VA-BRAVE eligibility criteria

\begin{tabular}{|c|c|}
\hline Inclusion criteria & Exclusion criteria \\
\hline $\begin{array}{l}\text { Use of opioids within } 30 \text { days prior to consent or within } 30 \text { days prior to } \\
\text { entry into a supervised setting } \\
\text { Meets DSM- } 5 \text { criteria for moderate to severe OUD } \\
\text { Referred to/seeking treatment for OUD } \\
\text { New episode of care, defined as requiring induction on buprenorphine OR } \\
\text { taking a form of prescribed medication treatment for OUD continuously } \\
\text { for < } 30 \text { days prior to consent }\end{array}$ & $\begin{array}{l}\text { Veterans }<18 \text { years of age } \\
\text { Females unwilling to practice an effective method of birth control for the } \\
\text { duration of the study } \\
\text { History of significant adverse effects from buprenorphine and/or naloxone } \\
\text { Recent suicidal or homicidal ideation or psychosis that requires hospitaliza- } \\
\text { tion } \\
\text { Unable or unwilling to provide consent } \\
\text { Meets criteria for current DSM-5 sedative hypnotic use disorder } \\
\text { Pending felony charges } \\
\text { Conditions which, in the judgement of the Local Site Investigator, make } \\
\text { it unlikely the patient can participate in or complete the 52-week active } \\
\text { phase of the study, including current moderate-to-severe COVID-19 symp- } \\
\text { toms with a risk of intubation or critical illness } \\
\text { Is actively participating in another interventional clinical trial for which a } \\
\text { waiver of dual-enrollment with VA-BRAVE has not been obtained }\end{array}$ \\
\hline
\end{tabular}

in VHA clinical settings. Participants who have moderate-to-severe OUD [Diagnostic Statistical manual-5th Edition (DSM-5)] [31] who are entering a new episode of care and who are 18 years or older are eligible. This includes participants with psychiatric, substance use and medical comorbidities that are typical in the VHA OUD population. Participants previously maintained on buprenorphine or other forms of MOUD are permitted to participate, but in order to be eligible for this trial they must be initiating buprenorphine as a new episode of care at the time of study enrollment $(<30$ consecutive days on MOUD treatment). As a real-world effectiveness trial, there are no restrictions in participation in psychosocial programs and other formal supports. Service utilization will be assessed as a secondary objective.

The exclusion criteria are also minimal to enhance generalizability, while maximizing patient safety. These include conditions (e.g., psychiatric conditions) requiring a higher level of care or medical conditions that preclude the use of buprenorphine. While participants are expected to have comorbid substance use disorders, whether to exclude participants using sedative hypnotics was carefully considered. Recent guidance from the American Society of Addiction Medicine (ASAM) [32] encourages treatment with buprenorphine even in those using sedative hypnotics since buprenorphine protects against risk of overdose from opioids. In this study, those participants at highest risk of combining medications, defined as those with a DSM-5 diagnosis of current sedative hypnotic use disorder are excluded.

Similarly, it is expected that participants will have medical comorbidities including hepatitis $B$ and $C$ and HIV. Only those participants for whom buprenorphine is medically contraindicated or who require intensive medical management (e.g., Childs-Pugh Class C cirrhosis) are excluded. Those with pending felony charges are also excluded due to likelihood of incarceration interfering with participation. In addition, in response to the COVID-19 pandemic, exclusion criteria exclude those with current moderate to severe COVID-19 symptoms who were at risk of intubation or critical illness. Those participants are able to join if they meet eligibility criteria after they recover from the acute phase of the infection.

Twenty geographically diverse sites were chosen across the United States with consideration for sites with facilities capable of supporting CSP research as well as those areas hard-hit by the opioid epidemic as shown in Fig. 2.

\section{Participant identification and consent}

This study is approved by the VA-Central Institutional Review Board (CIRB). Early identification, screening, and contact of patients seen by the medical center's MOUD clinic and substance use disorder specialty clinics, mental health clinics, primary care and sub-specialty medical clinics and inpatient units responsible for the evaluation and treatment of patients with likely or confirmed diagnoses of OUD are key to the study's success. CIRBapproved flyers and information sheets were developed to reach a broad and inclusive audience at local sites and result in patient-initiated contact with the local site study team.

Written consent for further screening and study participation is obtained in-person by an authorized member of the study team. Patients ineligible or unwilling to participate at any point in the recruitment process or who withdraw consent are referred for appropriate treatment via a warm handoff based upon consultation with the site study clinician.

\section{Devising clinically relevant treatment arms}

The goal of CSP 2014, VA-BRAVE, is to evaluate whether the long acting injectable buprenorphine is more effective 


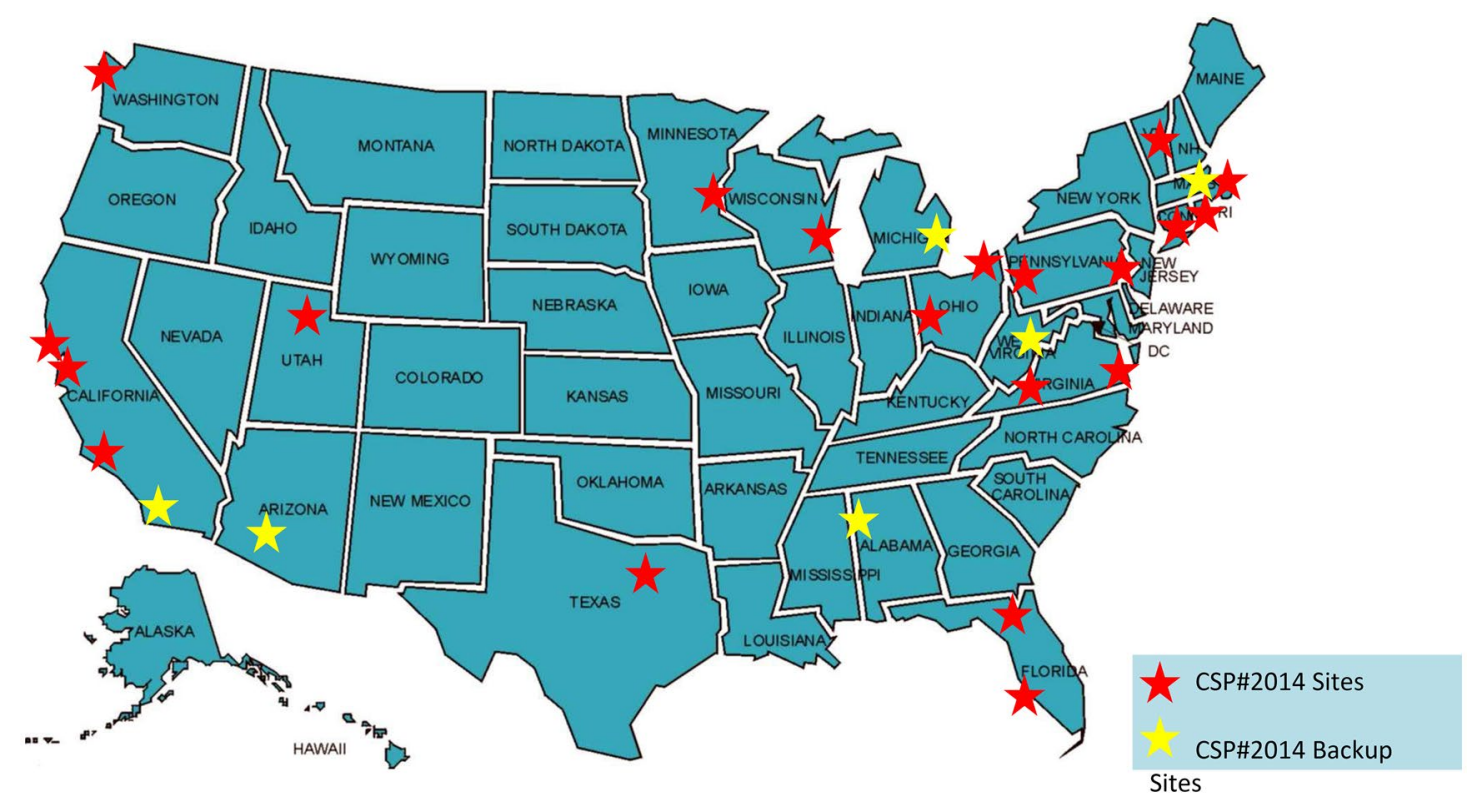

Fig. 2 CSP2014:VA-BRAVE map of study sites. Original active sites: Bay Pines, FL; Boston, MA; Cleveland, OH; Dallas, TX; Dayton, OH; Gainesville, FL; Hampton, VA; Long Beach, CA; Milwaukee, Wl; Palo Alto, CA; Philadelphia, PA; Pittsburgh, PA; Providence, Rl; Salem, VA; Salt Lake City, UT; San Francisco, CA; West Haven, CT; Seattle, WA; White River Junction, VT. Back-up sites: Bedford, MA; Detroit, Ml; Huntington, WV; Phoenix, AZ; San Diego, CA; Tuscaloosa, AL

than the current standard of care, sublingual buprenorphine, and whether its cost effectiveness merits its adoption. One design challenge was to isolate the route of administration as the independent variable and, in order to satisfy equipoise, careful consideration was taken in terms of induction processes, dose, and frequency of medication prescription.

Participants who meet eligibility criteria are initiated on SL-BUP/NLX as soon as clinically possible in accordance with good clinical practice. Buprenorphine is prescribed during an induction phase with SL-BUP/NLX starting at a dose of $2 \mathrm{mg}$ and then increased as needed for stabilization of opioid withdrawal symptoms. This induction procedure is consistent with clinical care as described in clinical practice guidelines [33] including SAMHSA TIP 63 practice guidelines [34]. The target maintenance dose is $16 \mathrm{mg}$ to $24 \mathrm{mg}$ titrated by day 3 , with room for clinical flexibility defined as not more than 30 days from induction. This study allows for rapid initiation of INJ-BUP in the service of getting patients stabilized on treatment as soon as possible. Potential participants who are already taking a form of buprenorphine for less than 30 days or who are in the process of being clinically inducted are also eligible for the study and bypass protocolized induction procedures. As soon as a maintenance dose is identified, the participant is ready for randomization.
Once reaching the target dose, eligible participants are randomized 1:1 to receive either continued daily SL-BUP/ NLX at the dose identified in the induction period or to receive monthly subcutaneous abdominal INJ-BUP with a target dose of $300 \mathrm{mg}$, with the option to use $100 \mathrm{mg}$ dose. Randomization is performed centrally by the CSP Clinical Research Pharmacy Coordinating Center (CSPCC) using an interactive web-based randomization program that allows for randomization of participants in real time by authorized study team members at each site who submit a randomization request form. The study participant is allocated to the appropriate treatment arm according to the randomization schema and a certificate is generated. The certificate is used by the local site investigator and research pharmacist to direct study drug dispensing. Participants are informed of their randomized treatment assignment during their visit, and prior to initiation of either SL-BUP/NLX or INJ-BUP.

This is an open label comparative effectiveness study so neither staff nor participants are blinded to treatment assignment.

\section{Study dose}

Study drug is prescribed for a treatment course totaling 52 weeks in either 28-day prescriptions of daily SL-BUP/ NLX (target dose 16-24 mg) or 28-day INJ-BUP (target dose $300 \mathrm{mg}$ ) injections, a formulation which provides 
steady blood level over 28-30 days. While two doses of INJ-BUP are available, $100 \mathrm{mg}$ and $300 \mathrm{mg}$, the $300 \mathrm{mg}$ dose was chosen as a target dose as it delivers a far more adequate steady state mean blood level and has been associated with better opioid abstinence outcomes than the $100 \mathrm{mg}$ dose for those who inject heroin [35]. The SL-BUP/NLX formulation used in this study is a sublingual film formulation across all sites. The dose range of $16-24 \mathrm{mg}$ is the standard dose recommended for clinical practice. The prescribing physician can make adjustments in dose for the SL-BUP/NLX following standard clinical practice. For those randomized to INJ-BUP, the $300 \mathrm{mg}$ dose is the target dose; however, the prescribing physician can lower the dose to $100 \mathrm{mg}$ after the first injection depending on patient preference or if clinically indicated (e.g., opioid agonist side effects). In order to satisfy equipoise, participants randomized to the SLBUP/NLX arm receive a 28-day take-home supply; while those in the INJ-BUP arm receive monthly INJ-BUP administered in the clinic at 28-day intervals. The study visits as depicted in Fig. 3 are identical between groups, and all participants regardless of their assigned treatment arm, receive a Medication Management (MM) session at each 28-day study visit (described below). Each site has a local site investigator and a study team; all study-related drugs are prescribed and administered by a clinician(s) (e.g., MD, NP, DO, PA, RN). Site investigators are mostly psychiatrists but also include primary care physicians; all are clinicians working in substance abuse clinics or in settings in which they are treating patients with opioid use disorder. Site investigators were chosen based on their experience with the patient population and with research (or with the availability of local research mentorship).

\section{Study length}

VA-BRAVE is a 52-week study that includes scheduled receipt of study medication and active follow-up. Many studies of OUD are limited to 12 or 24 weeks of active treatment; given that OUD can be chronic and relapsing, longer studies are needed to understand the clinical benefit of buprenorphine over time. This 52-week study will be better able to evaluate retention on study drug and opioid abstinence. In addition, there is an additional passive follow-up period, using the electronic medical record of up to 10 years (from date of first participant randomized), to assess longer-term patterns of service use including medications and hospitalizations.

\section{Retention}

Local staff will maintain participant engagement via periodic phone calls to remind them about visits, for checkins between visits, to follow-up on missed visits, and to obtain relevant study data if necessary. Participantprovided contact forms facilitate this outreach. Participants will be notified that those listed may be contacted but information regarding study participation will not be disclosed. Local staff will update this form as needed throughout the study. Participants will not be considered "lost to follow-up" until their week 52 visit, so there is always the opportunity to re-engage with the study after a lapse.

\section{Ethical design and care}

The study design has particular strengths that contribute to its scientific value and ensure ethical care. The choice of primary and secondary outcomes provides broad, patient-centered results, critical to the lives of Veterans seeking medication treatment for OUD. The

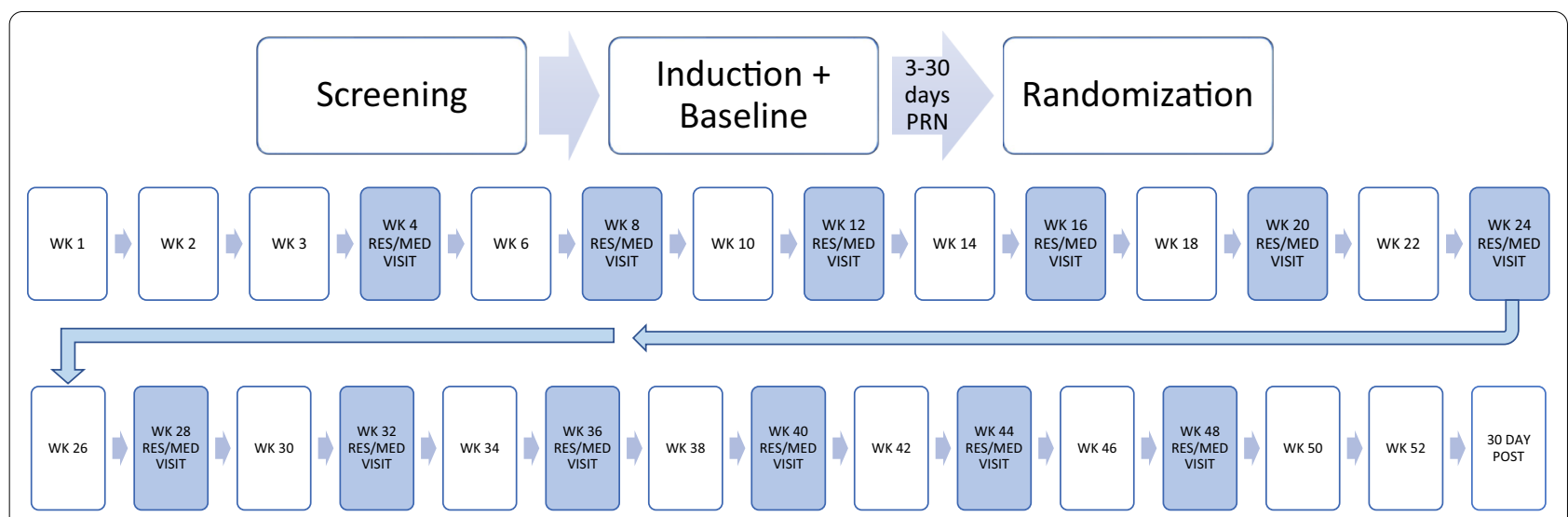

Fig. 3 CSP2014:VA-BRAVE timeline. PRN: as needed;WK: week; RES/Med VISIT: Research/Medication Administration Visit; 30 Day POST: 30 day post study safety monitoring period. *At each visit, research assessments are collected. For more information on the schedule of assessments, refer to Table 1 
study's exclusion criteria are minimal in order to make the results generalizable to a wide spectrum of Veterans seeking medication treatment for OUD (Table 1). Additionally, the induction phase preceding randomization follows established guidelines to engage participants in MOUD as early in treatment as possible.

Participants with OUD are at risk for opioid overdose. To mitigate this risk, study procedures ensure all participants receive Overdose Education and Naloxone Distribution (OEND) consistent with usual good clinical care practices for Veterans with OUD. In addition, all participants are provided Medication Management, a 15-min counseling session that recommends opioid and other substance use abstinence and adherence to MOUD.

\section{Outcomes}

One strength of this study is its use of two co-primary outcomes, retention and opioid abstinence, chosen because they are clinically meaningful, practical, patientcentered, and appropriate for a 52-week, large-scale comparative effectiveness trial.

Retention on treatment Retention on MOUD is a highly sensitive indicator of effective treatment, as discontinuation is strongly associated with recurrence to use of opioids, risk for overdose, transmission of bloodborne infectious diseases, and incarceration. Retention is defined as time from randomization to the first period of missed study-prescribed drug coverage lasting at least 4 weeks. This outcome is accurately measurable, unaffected by loss to follow-up, and highly indicative of clinical benefit.

Opioid abstinence reflects direct opioid use and will be indicated by self-report opioid abstinence using the systematic timeline follow-back (TLFB) [36] method and urine toxicology (UTOX) negative for opioids across 28 timepoints. The National Institute on Drug Abuse (NIDA)-funded Clinical Trials Network investigators have recommended using the method of self-report plus toxicology as a standard in substance use disorder trials.

Self-reported drug use is collected using the TLFB calendar method, in which study personnel record the participants' reported opioid use for each day since the last study visit. The TLFB is reliable and valid when used by trained interviewers and when there is no penalty for reporting use of drugs. The advantage to this method is that data are collected retrospectively to the last visit, so a missed appointment does not necessarily result in missing self-report data; limitations include reliance on self-report and lack of objective measurement. For that reason, a biological measure, with UTOX as standard, is also collected as an indicator of opioid abstinence. UTOX for this study is a UTOX-13 panel from a centralized lab, Redwood Toxicology, and collected at each study visit as indicated in Fig. 3 and the Schedule of Assessments in Table 2 and measures opioid drug use including oxycodone/noroxycodone, benzodiazepines, ethyl glucuronide, methadone, fentanyl, buprenorphine, tramadol, methylene-dioxy-methamphetamine (MDMA), amphetamines, cocaine metabolite, opiates, cannabinoids, and $\mathrm{THC} /$ creatinine ratio. UTOX screening is a routine part of buprenorphine maintenance treatment. UTOX screening methods have the issue of how to handle missing data [37]. For this trial, the conservative method that assumes missing UTOX is indicative of use is used (although participants may miss appointments for other reasons) and is consistent with most published research. Both the TLFB and UTOX data must be negative for opioid use to deem a participant abstinent.

\section{Secondary objectives}

Secondary objectives include determining whether the use of INJ-BUP is associated with better outcomes than SL-BUP/NLX in: opioid craving; use of other illicit substances and alcohol; preventing opioid overdose; reducing mental health symptoms (including depression, PTSD, and suicidality; homelessness); incarceration and criminal legal involvement; self-reported risky sexual and injection drug use behaviors and the incidence of new cases of HIV, HBV, and HCV. In addition, the total cost impact of INJ-BUP and cost-effectiveness from the perspective of VHA and of society will be evaluated. We will thus also assess non-VA service utilization, especially since the MISSION ACT [38], allows a Veteran to receive care from a community provider paid for by the VA. Table 2 summarizes the Schedule of Assessments including self-report and biological measures and their administration times. Data will also be captured to identify reasons for missed study injections as well as overall study visits.

\section{Special circumstances}

The following circumstances are anticipated for this population and considerations were made regarding study participation, procedures, and follow-up related to each.

\section{Incarceration}

Participants cannot be interviewed, have study procedures administered or receive study drug while incarcerated. However, if an incarceration episode has been concluded when discovered, and no immediate future risk of incarceration is present, then continuation of participation is reasonable. The study team may review the medical records of participants who are incarcerated. Participants with 'brief' incarceration periods, defined 
Table 2 CSP\#2014, VA-BRAVE study assessments

\begin{tabular}{|c|c|c|c|c|c|c|c|}
\hline Assessment & Screening & Baseline & Randomization & Weeks $1-3$ & Medication-research visits & $\begin{array}{l}\text { Non- } \\
\text { medication } \\
\text { visits }\end{array}$ & $\begin{array}{l}\text { End of study } \\
\text { visit (week } \\
52 \text { ) }\end{array}$ \\
\hline \multicolumn{8}{|l|}{ Biological assessments } \\
\hline Urine pregnancy test & $\sqrt{ }$ & $\sqrt{ }$ & $\sqrt{ }$ & & $\sqrt{ }$ & & $\sqrt{ }$ \\
\hline Physical exam & $\sqrt{ }$ & & & & Week 24 & & $\sqrt{ }$ \\
\hline $\begin{array}{l}\text { Liver and kidney function, } \\
\text { complete blood count (CBC) }\end{array}$ & $\sqrt{ }$ & & & & Weeks 4, 12, 24 & & $\sqrt{ }$ \\
\hline Electrocardiogram (EKG) & $\sqrt{ }$ & & & & Week $24^{*}$ & & \\
\hline Urine toxicology test & & $\sqrt{ }$ & & $\sqrt{ }$ & $\sqrt{ }$ & $\sqrt{ }$ & $\sqrt{ }$ \\
\hline $\begin{array}{l}\text { Blood HIV, hepatitis B (HBV), } \\
\text { and hepatitis } C(\mathrm{HCV})\end{array}$ & & $\sqrt{ }$ & & & Week 24 & & $\sqrt{ }$ \\
\hline $\begin{array}{l}\text { Blood buprenorphine and } \\
\text { norbuprenorphine }\end{array}$ & & $\sqrt{ }$ & & & Week 24 & & $\sqrt{ }$ \\
\hline \multicolumn{8}{|l|}{ Interviewer administered assessments } \\
\hline $\begin{array}{l}\text { The MINI International Neu- } \\
\text { ropsychic Interview (MINI) [39] }\end{array}$ & $\sqrt{ }$ & $\sqrt{ }$ & & & & & $\sqrt{ }$ \\
\hline $\begin{array}{l}\text { Clinical Opiate Withdrawal } \\
\text { Scale (COWS) [40] }\end{array}$ & & * & $\sqrt{ }$ & * & * & * & * \\
\hline $\begin{array}{l}\text { Clinical Institute Withdrawal Scale } \\
\text { (CIWA-Ar) [41] }\end{array}$ & $\sqrt{ }$ & * & * & * & * & * & * \\
\hline $\begin{array}{l}\text { Patient Health Questionnaire-9 } \\
\text { (PHQ-9, item } 9 \text { only) [42] }\end{array}$ & $\sqrt{ }$ & & & $\sqrt{ }$ & $\sqrt{ }$ & $\sqrt{ }$ & $\sqrt{ }$ \\
\hline Timeline follow-back [43] & & $\sqrt{ }$ & & $\sqrt{ }$ & $\sqrt{ }$ & $\sqrt{ }$ & $\sqrt{ }$ \\
\hline Concomitant medications & & $\sqrt{ }$ & & $\sqrt{ }$ & $\sqrt{ }$ & & $\sqrt{ }$ \\
\hline $\begin{array}{l}\text { Opioid craving and overdose } \\
\text { form }\end{array}$ & & $\sqrt{ }$ & & $\sqrt{ }$ & $\sqrt{ }$ & & $\sqrt{ }$ \\
\hline $\begin{array}{l}\text { PEG3 (pain, enjoyment, gen- } \\
\text { eral activity) }\end{array}$ & & $\sqrt{ }$ & & $\sqrt{ }$ & $\sqrt{ }$ & & $\sqrt{ }$ \\
\hline $\begin{array}{l}\text { Service utilization review form } \\
\text { (SURF) }\end{array}$ & & $\sqrt{ }$ & & & $\sqrt{ }$ & & $\sqrt{ }$ \\
\hline HIV risk behaviors & & $\sqrt{ }$ & & & Weeks $12,24,36$ & & $\sqrt{ }$ \\
\hline Criminal justice involvement & & $\sqrt{ }$ & & & Week 24 & & $\sqrt{ }$ \\
\hline $\begin{array}{l}\text { Alcohol Use Disorders Identifi- } \\
\text { cation Test (AUDIT) [44] }\end{array}$ & & $\sqrt{ }$ & & & & & $\sqrt{ }$ \\
\hline $\begin{array}{l}\text { Demographics and military } \\
\text { history }\end{array}$ & & $\sqrt{ }$ & & & & & \\
\hline $\begin{array}{l}\text { Modified substance use and } \\
\text { medical history }\end{array}$ & & $\sqrt{ }$ & & & & & \\
\hline $\begin{array}{l}\text { Columbia Suicide Severity Rat- } \\
\text { ing Scale (C-SSRS) [45] }\end{array}$ & & $\sqrt{ }$ & & & & & \\
\hline $\begin{array}{l}\text { Study Medication Adherence } \\
\text { Visual Analog Scale (VAS) }\end{array}$ & & & & $\sqrt{ }$ & $\sqrt{ }$ & & $\sqrt{ }$ \\
\hline Serious/adverse event & & * & * & * & * & * & * \\
\hline \multicolumn{8}{|l|}{ Self-report assessments } \\
\hline Veterans Rand-12 & & $\sqrt{ }$ & & & $\sqrt{ }$ & & $\sqrt{ }$ \\
\hline $\begin{array}{l}\text { Patient Health Questinnaire-9 } \\
\text { (PHQ 9) }\end{array}$ & & $\sqrt{ }$ & & & Weeks 12, 24, 36 & & $\sqrt{ }$ \\
\hline $\begin{array}{l}\text { Posttraumatic Stress Disorder } \\
\text { Checklist for DSM-5 (PCL-5) }\end{array}$ & & $\sqrt{ }$ & & & Weeks 12, 24, 36 & & $\sqrt{ }$ \\
\hline COVID-19 Questionnaire & & $\sqrt{ }$ & & & Weeks $12,24,36$ & & $\sqrt{ }$ \\
\hline
\end{tabular}

$\sqrt{ }=$ collected at each timepoint; ${ }^{*}=$ collected only when necessary; please note some assessments are collected only during weeks indicated in the table 
as a period of less than the remainder of the participant's 52-week study period, may be eligible to continue study participation if they so choose after they are released back to the community.

\section{Non-fatal and fatal accidental overdose}

Information regarding accidental drug poisoning (i.e., overdose) will be obtained and tracked via participant self-report, naloxone distribution records, hospital records both within and outside VHA, as well as CDC accidental drug poisoning data, if possible. If a participant experiences non-fatal accidental drug poisoning, this would not necessarily impact eligibility.

\section{Newly diagnosed HIV/HBV/HCV infections}

Information regarding $\mathrm{HIV} / \mathrm{HBV} / \mathrm{HCV}$ will be obtained and tracked via participant self-report, $\mathrm{HIV} / \mathrm{HBV} / \mathrm{HCV}$ blood tests at periodic study visits (see Table 2), and hospital records both within and outside VHA. If a participant has a newly diagnosed HIV/HBV/HCV infection, this would not necessarily impact eligibility or continued study participation.

\section{Pregnancy}

Urine pregnancy testing will be conducted on all Veterans of childbearing potential at screening, induction, baseline, and randomization as well as all follow-up visits during which study medication is prescribed/administered as denoted in Table 2. For these female Veterans, pregnancy, breastfeeding, and/or failure to practice an effective method of birth control for the duration of participation in the study are exclusion criteria (Table 1). In the event of a positive pregnancy test, the participant will be referred to clinical care and study-related treatment will stop.

\section{SARS COV2 (COVID-19)}

As the clinical research landscape is ever-changing during the coronavirus disease (COVID-19) pandemic, researchers must adapt in making decisions about implementing the research protocol in clinical trials. Regulations governing buprenorphine treatment were relaxed in response to the COVID pandemic to allow initiation and maintenance of buprenorphine by telemedicine without in person visits. However, due to the needs of the research to collect urine in person and the relatively low likelihood of patients presenting with active COVID infection, some in person visits were deemed necessary.

Study procedures were modified to be conducted in a manner that protects both staff and participants from COVID-19 by minimizing any risk for inadvertent transmission and by limiting exposure time. All study drug initiations, provisions of SL-BUP/NLX prescriptions, and injections of INJ-BUP are performed in person.

If potential participants are COVID-19 positive or in COVID-19 isolation, they may be given information about the study via telephone/telehealth, but may not be consented, as these procedures must occur in-person to assess and ensure informed consent. However, eligibility may be reconsidered when the patient has clinically improved.

Enrolled participants are screened per standard of care at each participating site for COVID-19 symptoms and exposure; research procedures may continue on a full face-to-face or limited face-to-face basis for those who screen negative. If an already enrolled participant is under quarantine or isolation orders, they may not be seen in person for study-related procedures. Study procedures may be rescheduled and/or conducted via telephone/telehealth. Study visit assessments and procedures that may be conducted via telehealth/telephone have been identified and include predominantly selfreport measures. In order to capture information about COVID-19 and its effect on drug use, a COVID-19 questionnaire has been added to the protocol for administration at baseline and every 3 months.

\section{Analytic plan}

The primary objective is to determine if long-acting injectable formulation of buprenorphine is superior to the sublingual formulation. There are two complementary primary endpoints: retention on study assigned MOUD and opioid abstinence. For the retention outcome, a time-to-event analysis using a two-sided logrank test will compare the treatments. For the abstinence outcome, the mean number of opioid free UTOX screens in conjunction with self-reported abstinence at 28 distinct time points in each treatment arm will be compared by a Student $\mathrm{t}$-test. Both measures must be negative for classification as abstinent. We will accrue over the follow-up period the total count of such self-report consistent opioid free urine tests. In secondary analyses UTOX samples obtained during study visits will be compared to self-reports of opioid use. All secondary analyses are exploratory and therefore not powered. All analyses of these outcome variables will be done with a single predictor, formulation (simple or one variable), or with formulation and a stock of covariates (multivariable) that predict the outcome. Predictive variable will be used for secondary analyses in which the outcomes used in the primary analyses will be assessed based upon explanatory variables that might affect treatment group outcomes between the two intervention groups. 
All clinical data and study documents will be monitored by the CSP Coordinating Center (CSPCC) using an electronic data capture and clinical trial management system and follow existing regulations and standard operating procedures in handling study and participant information and used in VA Cooperative Studies.

\section{Sample size and power analysis}

The sample size estimates primarily arise from the assumption of an overall at least $40 \%$ retention after 52 weeks. The study treatment retention times will be compared using a log-rank test, assuming that for the alternative hypothesis, the hazard ratio (injected over sublingual) is 0.74 or smaller. The two-sided hypothesis also rejects the null hypothesis if the hazard ratio is 1.36 or larger. For the comparison of abstinence, using the t-test, the alternative hypothesis posits a difference in proportions of $10 \%$ (a difference of 1.5 if mean counts are about 15). Under these assumptions, with a total size of 952 participants, this study has overall $90 \%$ power to reject both null hypotheses. Each test assumes a Type I error of $2.4 \%$ (where the interim uses $0.1 \%$ ). This assumes no participants drop out (they are treatment failures), but that follow-up time ends when the participant dies or has a long-term hospitalization, is institutionalized, or incarcerated.

\section{Discussion}

CSP \#2014, VA-BRAVE, was developed soon after a new formulation of injectable buprenorphine became available for use. The study was designed as a large-scale comparative effectiveness trial designed to enroll patients with moderate to severe OUD seeking medication treatment who are enrolled in care in the VHA. The inclusion criteria are considerably broad to allow enrollment of Veterans with comorbid conditions, the co-primary outcomes of retention and abstinence were chosen as meaningful clinical measures of success, the secondary outcomes including health services and the long duration of follow-up were included to collect meaningful clinical information on the use of injectable medications in OUD. The study was designed with consideration of special populations of VHA patients with OUD, i.e., with comorbid psychiatric disorders, high rates of criminal justice involvement, infectious disease comorbidity, and/or high rates of overdose. Out of necessity, special considerations for research during the COVID-19 pandemic were also included.

Conducting this study in the VHA deserves special mention. The VHA is the largest healthcare provider in the country and currently serves approximately 81,000 Veterans with OUD annually and these numbers have continued to grow. The VA is a national system, with an electronic medical record allowing for capture of both medical and health services information, providing integrated clinical care. The robust research infrastructure of VA, and the Cooperative Studies Program specifically includes pharmacy support, data management, etc. The VA Cooperative Studies Program also has a strong track record in minority recruitment, particularly in studies of conditions prevalent in black populations [46]. Of note, this is the first CSP study to focus on OUD. Providing MOUD for OUD is a key objective for the VHA [33, 47]. This new monthly injectable formulation of buprenorphine currently represents a promising new improvement in treatment of OUD.

There are some limitations associated with this study design. In order for equipoise, all participants who are randomized to the SL-BUP/NLX arm receive a 28 -day supply of medication and have an identical number of research visits as those who are randomized to the monthly INJ-BUP arm. Similarly, while INJ-BUP may have other advantages over SL-BUP/NLX such as in preventing diversion, this study will not be evaluating diversion. Finally, there is now a new injectable formulation of buprenorphine available that was not FDA approved when this study was funded [48, 49]. The question remains whether conclusions from this study will be generalizable to all injectable formulations.

\section{Conclusion}

Positive findings in this trial could lead to widespread adoption by clinicians, that could reduce opioid use among Veterans, likely improve Veterans' medical, psychological, and social outcomes, and undoubtedly save lives. If injectable buprenorphine is not superior to sublingual buprenorphine/naloxone, its use can be formally limited through the VA formulary for use in special circumstances. This study's findings, positive or negative, would also be applicable to much of the non-VA opioid use disorder treatment community and would contribute substantially to the nation's ability to respond effectively to our current opioid epidemic.

\footnotetext{
Abbreviations

ASAM: American Society of Addiction Medicine; AUDIT: Alcohol Use Disorders Identification Test; CBC: Complete blood count; CIRB: Central Institutional Review Board; CIWA-Ar: Clinical Institute Withdrawal Scale; COWS: Clinical Opiate Withdrawal Scale; CSP: Cooperative Studies Program; CSPCC: Cooperative Studies Program Coordinating Center; C-SSRS: Columbia Suicide Severity Rating Scale; DO: Doctor of osteopathic medicine; EKG: Electrocardiogram; HBV: Hepatitis B; HCV: Hepatitis C; HIV: Human immunodeficiency virus; IDEAS: Informatics, Decision-Enhancement, and Analytic Sciences Center; INJ-BUP: Injectable monthly buprenorphine; MD: Doctor of medicine; MDMA: Methylene-dioxy-methamphetamine; MINI: The Mini International Neuropsychic Interview; MM: Medication management; MOUD: Medication treatments for OUD; NP: Nurse Practitioner; OEND: Overdose Education Naloxone Distribution; OUD: Opioid use disorder; PA: Physician Assistant; PARCKA: Program
} 
for Addiction Research, Clinical Care, Knowledge and Advocacy; PCL-5: Posttraumatic Stress Disorder Checklist for DSM-5; PEG3: Pain, enjoyment, genera activity; PHQ-9: Patient Health Questionnaire-9; PTSD: Post-traumatic stress disorder; SL-BUP/NLX: Sublingual buprenorphine/naloxone; SURF: Service utilization review form; RCT: Randomized control trial; RN: Registered nurse; TLFB: Timeline follow-back; UTOX: Urine toxicology; NIDA: National Institute on Drug Abuse; VA: Veterans Affairs; VA-BRAVE: Buprenorphine for Treating Opioid Use Disorder in Veterans; VAS: Visual Analog Scale; VHA: Veterans Healthcare Administration; XR-NTX: Extended-release injectable formulation.

\section{Acknowledgements}

VA-Cooperative Studies Scientific Evaluation Committee (CSSEC), Melynn Nuite, Alysa Shanin, Anne Slaven, Chris Donnelly and Diana DeNegre. CSP \#2014 Planning Committee-Ismene Petrakis, Sandra A. Springer, Cynthia Davis, Elizabeth Ralevski, Lucy Gu, Robert Lew, John Hermos, Alexa Goldberg, Robert Ringer, Ryan Ferguson. CSP \#2104 External Advisors to the Planning Committee—Adam J. Gordon, Thomas R. Kosten, Edward V. Nunes, Robert Rosenheck, Andrew J. Saxon. CSP\#2014 External Executive Committee-John Hermos, Adam J. Gordon, Thomas R. Kosten, Edward V. Nunes, Robert Rosenheck, Andrew J. Saxon, Robert Swift.

\section{Disclaimer}

The views expressed do not necessarily represent the views of the Department of Veterans Affairs or the US Government.

\section{Authors' contributions}

All authors contributed to the design of this study and contributed in the writing of the manuscript. All authors read and approved the final manuscript.

\section{Funding}

Supported entirely by the Cooperative Studies Program, Department of Veterans Affairs, Office of Research and Development.

\section{Availability of data and materials}

Not applicable.

\section{Declarations}

\section{Ethics approval and consent to participate}

This study is approved by the VA-Central Institutional Review Board (CIRB).

\section{Consent for publication}

Not applicable.

\section{Competing interests}

Authors S. Springer and I. Petrakis have received consultation honoraria and have received study drug donated in-kind from Alkermes Inc; Author S. Springer has received study drug donated in-kind from Indivior Inc. for a NIDA-funded study. TR Kosten has received consultation honoraria from Alkermes Inc. Author A. Saxon received consultation honorarium from Indivior, Inc., received consultation honorarium and travel support from Alkermes, Inc., and royalties from UpToDate, Inc. Author Adam J. Gordon receive royalties from UpToDate, Inc., for an online chapter unrelated to this manuscript.

\footnotetext{
Author details

'Department of Psychiatry, Veterans Affairs Connecticut Healthcare System, West Haven, CT, USA. '2 Department of Psychiatry, Yale School of Medicine, New Haven, CT, USA. ${ }^{3}$ Section of Infectious Disease, Department of Internal Medicine, Veterans Affairs Connecticut Healthcare System, West Haven, CT, USA. ${ }^{4}$ Section of Infectious Disease, Department of Internal Medicine, Yale School of Medicine, New Haven, CT, USA. ${ }^{5}$ US Department of Veteran Affairs, Cooperative Studies Program Coordinating Center (CSPCC), Boston, MA, USA. ${ }^{6}$ Department of Psychiatry, Harvard Medical School, Boston, MA, USA. ${ }^{7}$ Center for Healthcare Organization and Implementation Research, VA Boston Healthcare System, Jamaica Plain, Boston, MA, USA. ${ }^{8}$ Department of Public Health, Boston University, Boston, MA, USA. ${ }^{9}$ Section of General Internal Medicine, Department of Medicine, School of Medicine, Boston University, Boston, MA, USA. ${ }^{10}$ Department of Internal Medicine, VA Boston Healthcare System, Jamaica Plain, Boston, MA, USA. ${ }^{1}$ Informatics, Decision-Enhancement, and Analytic Sciences (IDEAS) Center, VA Salt Lake City Health Care System, Salt
}

Lake City, UT, USA. ${ }^{12}$ Division of Epidemiology, Department of Internal Medicine, Program for Addiction Research, Clinical Care, Knowledge and Advocacy (PARCKA), University of Utah School of Medicine, Salt Lake City, UT, USA. ${ }^{13}$ Department of Psychiatry, Michael E. DeBakey Veterans Affairs Medical Center, Houston, TX, USA. ${ }^{14}$ Department of Psychiatry, Baylor College of Medicine, Houston, TX, USA. ${ }^{15}$ Department of Psychiatry, Columbia University Medical Center, New York, NY, USA. ${ }^{16}$ Center of Excellence in Substance Addiction Treatment and Education, VA Puget Sound Health Care System, Seattle, WA, USA. ${ }^{17}$ Department of Psychiatry and Behavioral Sciences, University of Washington School of Medicine, Seattle, WA, USA. ${ }^{18}$ Providence Veterans Affairs Medical Center, Providence, RI, USA. ${ }^{19}$ Department of Psychiatry and Human Behavior, Center for Alcohol and Addiction Studies, Brown University Warren Alpert Medical School, Providence, RI, USA. ${ }^{20}$ US Department of Veteran Affairs, Cooperative Studies Program Clinical Research Pharmacy Coordinating Center, Albuquerque, NM, USA.

Received: 27 April 2021 Accepted: 10 January 2022

Published online: 31 January 2022

\section{References}

1. O'Donnell JK, Gladden RM, Seth P. Trends in deaths involving heroin and synthetic opioids excluding methadone, and law enforcement drug product reports, by census region-United States, 2006-2015. MMWR Morb Mortal Wkly Rep. 2017;66:897-903. https://doi.org/10.15585/mmwr. mm6634a2.

2. Han B, Compton WM, Jones CM, et al. Nonmedical prescription opioid use and use disorders among adults aged 18 through 64 years in the United States, 2003-2013. JAMA. 2015;314:1468-78. https://doi.org/10. 1001/jama.2015.11859.

3. Hedegaard H, Warner M, Miniño AM. Drug overdose deaths in the United States, 1999-2016. NCHS Data Brief. 2017:1-8.

4. Lin L, Peltzman T, McCarthy JF, et al. Changing trends in opioid overdose deaths and prescription opioid receipt among veterans. Am J Prev Med. 2019;57:106-10. https://doi.org/10.1016/j.amepre.2019.01.016.

5. Wyse JJ, Gordon AJ, Dobscha SK, et al. Medications for opioid use disorder in the department of veterans affairs (VA) health care system: historical perspective, lessons learned and next steps. Subst Abus. 2018. https://doi.org/10.1080/08897077.2018.1452327.

6. Gellad WF, Good CB, Shulkin DJ. Addressing the opioid epidemic in the United States: lessons from the department of veterans affairs. JAMA Internal Med. 2017;177:611-2. https://doi.org/10.1001/jamainternmed. 2017.0147.

7. Ahmad F, Escobedo L, Rossen L, et al. Provisional drug overdose death counts. Hyattsville: National Center for Health Statistics; 2020.

8. Wilson N, Kariisa M, Seth P, et al. Drug and opioid-involved overdose deaths_United States, 2017-2018. MMWR Morb Mortal Wkly Rep. 2020;69:290-7. https://doi.org/10.15585/mmwr.mm691 1a4.

9. Haley DF, Saitz R. The opioid epidemic during the COVID-19 pandemic. JAMA. 2020. https://doi.org/10.1001/jama.2020.18543.

10. McCance-Katz E. SAMHSA/HHS: an update on the opioid crisis. Rockville: SAMHSA; 2018

11. Gordon AJ, Drexler K, Hawkins EJ, et al. Stepped care for opioid use disorder train the trainer (SCOUTT) initiative: expanding access to medication treatment for opioid use disorder within Veterans Health Administration facilities. Subst Abus. 2020;41:275-82. https://doi.org/10.1080/08897077. 2020.1787299.

12. Iheanacho T, Stefanovics E, Rosenheck R. Opioid use disorder and homelessness in the Veterans Health Administration: the challenge of multimorbidity. J Opioid Manag. 2018;14:171-82. https://doi.org/10. 5055/jom.2018.0447.

13. Schuckit MA. Treatment of opioid-use disorders. N Engl J Med. 2016;375:357-68. https://doi.org/10.1056/NEJMra1604339.

14. SAMSHSA. Buprenorphine. Substance Abuse and Mental Health Services Administration: Rockville; 2018.

15. Amato L, Davoli M, Perucci CA, et al. An overview of systematic reviews of the effectiveness of opiate maintenance therapies: available evidence to inform clinical practice and research. J Subst Abuse Treat. 2005;28:321-9. https://doi.org/10.1016/j.jsat.2005.02.007. 
16. Global Commission on Drug Policy. Taking control: pathways to drug policies that work. https://www.globalcommissionondrugs.org/reports/ taking-control-pathways-to-drug-policies-that-work (2014).

17. World Health Organization. WHO/UNODC/UNAIDS position paper: substitution maintenance therapy in the management of opioid dependence and HIV/AIDS prevention. Geneva: World Health Organization, United Nations Office on Drugs and Crimes, UNAIDS; 2004.

18. Wong KH, Lee SS, Lim WL, et al. Adherence to methadone is associated with a lower level of HIV-related risk behaviors in drug users. J Subst Abus Treat. 2003;24:233-9. https://doi.org/10.1016/s0740-5472(03)00029-1.

19. Timko C, Schultz NR, Cucciare MA, et al. Retention in medication-assisted treatment for opiate dependence: a systematic review. J Addict Dis. 2016:35:22-35. https://doi.org/10.1080/10550887.2016.1100960.

20. Morgan JR, Schackman BR, Leff JA, et al. Injectable naltrexone, oral naltrexone, and buprenorphine utilization and discontinuation among individuals treated for opioid use disorder in a United States commercially insured population. J Subst Abus Treat. 2018;85:90-6. https://doi. org/10.1016/j.jsat.2017.07.001.

21. O'Connor AM, Cousins G, Durand L, et al. Retention of patients in opioid substitution treatment: a systematic review. PLOS ONE. 2020;15: e0232086. https://doi.org/10.1371/journal.pone.0232086.

22. Manhapra A, Petrakis I, Rosenheck R. Three-year retention in buprenorphine treatment for opioid use disorder nationally in the Veterans Health Administration. Am J Addict. 2017;26:572-80. https://doi.org/10.1111/ ajad. 12553.

23. Maddux JF, Desmond DP. Ten-year follow-up after admission to methadone maintenance. Am J Drug Alcohol Abus. 1992;18:289-303.

24. Stimmel B, Goldberg J, Rotkopf E, et al. Ability to remain abstinent after methadone detoxification. A six-year study. JAMA 1977; 237: 1216-1220. 1977/03/21.

25. Manhapra A, Rosenheck R, Fiellin DA. Opioid substitution treatment is linked to reduced risk of death in opioid use disorder. BMJ. 2017;357: j1947. https://doi.org/10.1136/bmj.j1947.

26. Schwarz R, Zelenev A, Bruce RD, et al. Retention on buprenorphine treatment reduces emergency department utilization, but not hospitalization, among treatment-seeking patients with opioid dependence. J Subst Abus Treat. 2012;43:451-7. https://doi.org/10.1016/j.jsat.2012.03.008.

27. Springer SA, Chen S, Altice FL. Improved HIV and substance abuse treatment outcomes for released HIV-infected prisoners: the impact of buprenorphine treatment. J Urban Health. 2010;87:592-602. https://doi. org/10.1007/s11524-010-9438-4.

28. Springer SA, Qiu J, Saber-Tehrani AS, et al. Retention on buprenorphine is associated with high levels of maximal viral suppression among HIVinfected opioid dependent released prisoners. PLoS ONE. 2012;7: e38335. https://doi.org/10.1371/journal.pone.0038335.

29. Haight BR, Learned SM, Laffont CM, et al. Efficacy and safety of a monthly buprenorphine depot injection for opioid use disorder: a multicentre, randomised, double-blind, placebo-controlled, phase 3 trial. Lancet. 2019;393:778-90. https://doi.org/10.1016/s0140-6736(18)32259-1.

30. Rioux J, Cuellar N, Oliver J, et al. Evaluating retention rates in a medication-assisted treatment program. J Nurse Pract. 2020;16:e149-51. https:// doi.org/10.1016/j.nurpra.2020.06.028.

31. APA. Diagnostic and statistical manual of mental disorders. 5th ed. Washington, D.C: American Psychiatric Association; 2013.

32. ASAM. The ASAM national practice guideline for the treatment of opioid use disorder: 2020 focused update: Erratum. J Addict Med. 2020;14:1-91.

33. The Management of Substance Abuse Use Disorders Working Group. In: The Office of Quality and Safety VA Washington DC and Quality Management Division United States Army MEDCOM, editors. VA/DoD clinical practice guidelines for management of substance use disorders (SUD). 2015; Washington DC. p. 1-169.

34. SAMHSA SAaMHSA. Medications for opioid use disorder. In: Administration SAaMHS, editor. Treatment improvement protocol (TIP) series 63. Rockville: Substance Abuse and Mental Health Services Administration; 2018.

35. Slough I. INDIVIOR PLC results from the phase 3 pivotal study of RBP-6000 buprenorphine monthly depot for the treatment of opioid use disorder. In: Conference on problem of drug dependence (CPDD). Montreal, Canada; 2017
36. Sobell LC, Brown J, Leo Gl, et al. The reliability of the alcohol timeline followback when administered by telephone and by computer. Drug Alcohol Depend. 1996:42:49-54.

37. Biondi BE, Zheng $X$, Frank CA, et al. A literature review examining primary outcomes of medication treatment studies for opioid use disorder: what outcome should be used to measure opioid treatment success? Am J Addict. 2020;29:249-67. https://doi.org/10.1111/ajad.13051.

38. Veterans Administration. VA MISSION Act. https://missionact.va.gov/. (2020).

39. Sheehan DV, Lecrubier Y, Sheehan KH, et al. The mini-international neuropsychiatric interview (MINI): the development and validation of a structured diagnostic psychiatric interview for DSM-IV and ICD-10. J Clin Psychiatry. 1998;59(Suppl 20):22-33 (quiz 34-57).

40. Wesson DR, Ling W. The clinical opiate withdrawal scale (COWS). J Psychoact Drugs. 2003;35:253-9.

41. Sullivan J, Sykora K, Schneiderman J, et al. Assessment of alcohol withdrawal: the revised clinical institute withdrawal assessment for alcohol scale (CIWA-Ar). Br J Addict. 1989:84:1353-7.

42. Kroenke K, Spitzer RL, Williams JB. The PHQ-9: validity of a brief depression severity measure. J Gen Intern Med. 2001;16:606-13.

43. Sobell LC, Sobell MB. Timeline follow-back: a technique for assessing selfreported alcohol consumption. In: Litten RZ, Allen J, editors. Measuring alcohol consumption: psychosoical and biological methods. Totowa: Humana Press; 1992. p. 41-72.

44. Bohn MJ, Babor TF, Kranzler HR. The alcohol use disorders identification test (AUDIT): validation of a screening instrument for use in medical settings. J Stud Alcohol. 1995:56:423-32.

45. Posner K, Brent D, Lucas C, et al. Columbia-suicide severity rating scale (C-SSRS). New York: Columbia Univeristy Medical Center; 2008.

46. Oddone EZ, Olsen MK, Lindquist JH, et al. Enrollment in clinical trials according to patients race: experience from the VA cooperative studies program (1975-2000). Control Clin Trials. 2004;25:378-87. https://doi.org/ 10.1016/j.cct.2004.05.001.

47. The White House. Fact sheet: President Obama proposes $\$ 1.1$ billion in new funding to address the prescription opioid abuse and heroin use epidemic. Washington, D.C: The White House; 2016.

48. Lofwall MR, Walsh SL, Nunes EV, et al. Weekly and monthly subcutaneous buprenorphine depot formulations vs daily sublingual buprenorphine with naloxone for treatment of opioid use disorder: a randomized clinical trial. JAMA Internal Med. 2018;178:764-73. https://doi.org/10.1001/jamai nternmed.2018.1052.

49. Frost M, Bailey GL, Lintzeris N, et al. Long-term safety of a weekly and monthly subcutaneous buprenorphine depot (CAM2038) in the treatment of adult out-patients with opioid use disorder. Addiction. 2019;114:1416-26. https://doi.org/10.1111/add.14636.

\section{Publisher's Note}

Springer Nature remains neutral with regard to jurisdictional claims in published maps and institutional affiliations.

Ready to submit your research? Choose BMC and benefit from:

- fast, convenient online submission

- thorough peer review by experienced researchers in your field

- rapid publication on acceptance

- support for research data, including large and complex data types

- gold Open Access which fosters wider collaboration and increased citations

- maximum visibility for your research: over 100M website views per year

At BMC, research is always in progress.

Learn more biomedcentral.com/submissions 